\title{
Proposal for GPD studies at COMPASS
}

\author{
E. Burtin, on behalf of the COMPASS collaboration
}

\author{
CEA - Centre de Saclay \\ Irfu, Service de Physique Nucléaire \\ F-91191 Gif-sur Yvette Cedex, France \\ email=eburtin@cea.fr
}

\begin{abstract}
The study of nucleon structure through Generalised Parton Distributions (GPD) is one major part of the future COMPASS-II physics program [1] and can be performed using exclusive reactions like Deeply Virtual Compton Scattering (DVCS) and Meson Production. The high energy of the muon beam allows to measure the $x_{B}$-dependence of the $t$-slope of the DVCS cross section. The use of positive and negative polarised muon beams allows to determine the Beam Charge and Spin Difference of the DVCS cross sections to access the real part of the Compton form factor related to the dominant GPD $\mathrm{H}$. The sensitivity of both measurements is examined and confronted to existing models or global fits of the data. Preliminary beam test data were analyzed and demonstrated the feasibility of the identification of the DVCS reaction using the COMPASS experimental set-up.
\end{abstract}

Keywords: Nucleon Structure, Generalised Parton Distributions, Deeply Virtual Compton Scattering, Exclusive reactions PACS: -

\section{CONTENTS}

Kinematic Domain accessible at COMPASS

DVCS Measurement with polarized $\mu^{+}$and $\mu^{-}$beams 1

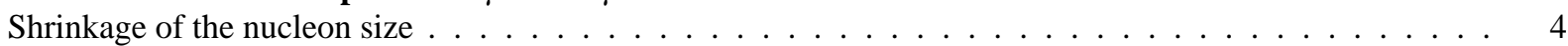

Beam Charge and Spin Asymmetry and sensitivity to models . . . . . . . . . . . . . . . . . . 5

Observation of exclusive photon production in 2008 and 2009 test beam data 5

\section{KINEMATIC DOMAIN ACCESSIBLE AT COMPASS}

The COMPASS experiment is located on the unique high-energy $(200 \mathrm{GeV})$ and highly polarized $\mu^{ \pm}$beam line of the CERN SPS and uses a high resolution forward spectrometer in conjunction with a fixed target (unpolarized or longitudinally or transversely polarized). By installing a recoil proton detector around the target to ensure exclusivity of Deeply Virtual Compton Scattering (DVCS) and Deeply Virtual Meson Production (DVMP) events, it could be converted into a facility measuring exclusive reactions within a kinematic subspace ranging from $x \sim 0.005$ to $\sim 0.1$, which cannot be explored by any other existing or planned facility in the near future. Figure 1 displays the kinematic domain of the fixed-target experiments COMPASS, HERMES and JLab. These domains are indicated between the dotted lines defined by the maximum energy of the facility and the curve $\mathrm{W}>2 \mathrm{GeV}$, i.e. above the resonance domain. COMPASS would thus explore the uncharted $x$ domain between those of H1 and ZEUS at the HERA collider and of fixed-target experiments as HERMES and the planned $12 \mathrm{GeV}$ extension of the JLab accelerator.

\section{DVCS MEASUREMENT WITH POLARIZED $\mu^{+}$AND $\mu^{-}$BEAMS}

DVCS is considered to be the theoretically cleanest of the experimentally accessible processes to measure GPDs because effects of next-to-leading order and subleading twist are under theoretical control [2]. The competing BetheHeitler $(\mathrm{BH})$ process which is elastic lepton-nucleon scattering with a hard photon emitted by either the incoming or 


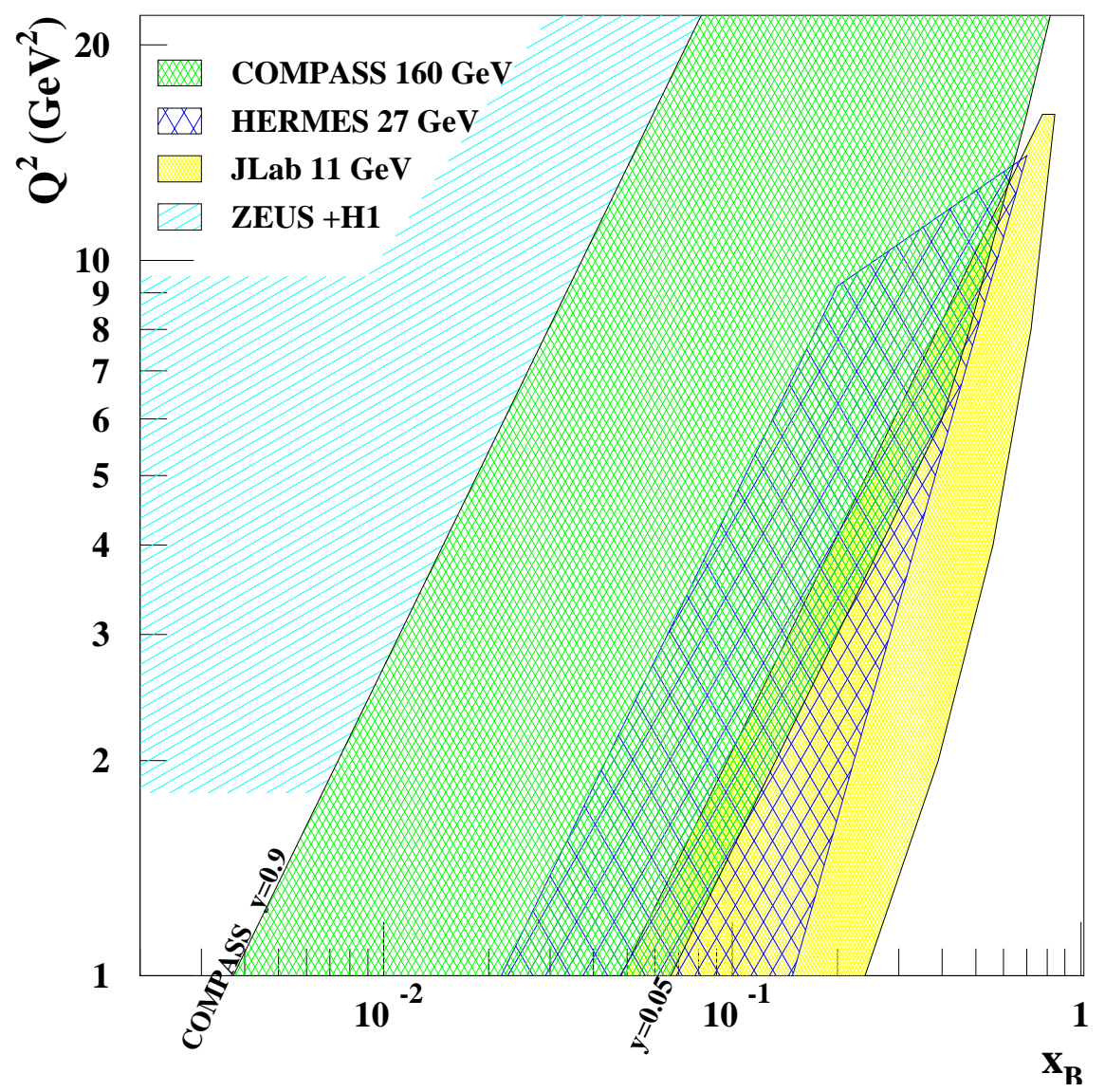

FIGURE 1. Kinematic domains for measurements of hard exclusive processes shown for the COMPASS (green area enclosed by the lines $y=0.9$ and $y=0.05)$, HERMES and JLab fixed-target experiments and the HERA collider experiments H1 and ZEUS.

outgoing lepton, has a final state identical to that of DVCS so that both processes interfere at the level of amplitudes $\mathscr{A}:$

$$
d \sigma(\mu \mathrm{N} \rightarrow \mu \mathrm{N} \gamma) \propto\left|\mathscr{A}_{B H}\right|^{2}+\left|\mathscr{A}_{D V C S}\right|^{2}+\underbrace{\mathscr{A}_{B H} \mathscr{A}_{D V C S}^{*}+\mathscr{A}_{B H}^{*} \mathscr{A}_{D V C S}}_{I}
$$

The Bethe-Heitler amplitude can be calculated using QED and elastic form factors measurements. The collection of almost pure BH events at small $x$ allows one to get an excellent reference yield and to control accurately the efficiency of the detection. In contrast the collection of almost pure DVCS events at larger $x$ will allow for the measurement of the $x$-dependence of the t-slope of the cross section which is related to the tomographic partonic image of the nucleon. In the intermediate domain, the DVCS contribution will be enhanced by the BH process through their interference. COMPASS is presently the only facility to provide polarized leptons with either charge: polarized $\mu^{+}$and $\mu^{-}$beams. As the $\mathrm{BH}$ is independent of charge and polarization, this contribution can be removed by subtracting 2 separate measurements obtained for the two beam charges. Moreover the natural polarization of the muon beam produced from pion decay changes sign when the beam charge is reversed and the different topologies of $\mu^{+}$and $\mu^{-}$, polarized with opposite direction, allow one to select only the real part or the imaginary part of the complex amplitude of DVCS. COMPASS offers the advantage to provide various kinematic domains where either BH or DVCS dominate (see Fig. 2).

For the muo-production of real photons off an unpolarized proton target, the differential cross section can be written as :

$$
\frac{d^{4} \sigma(\mu p \rightarrow \mu p \gamma)}{d x_{B} d Q^{2} d \mathrm{t} d \phi}=d \sigma^{B H}+\left[d \sigma_{\text {unpol }}^{D V C S}+P_{\mu} d \sigma_{\text {pol }}^{D V S C}\right]+e_{\mu}\left[\operatorname{Re} I+P_{\mu} \operatorname{Im} I\right]
$$



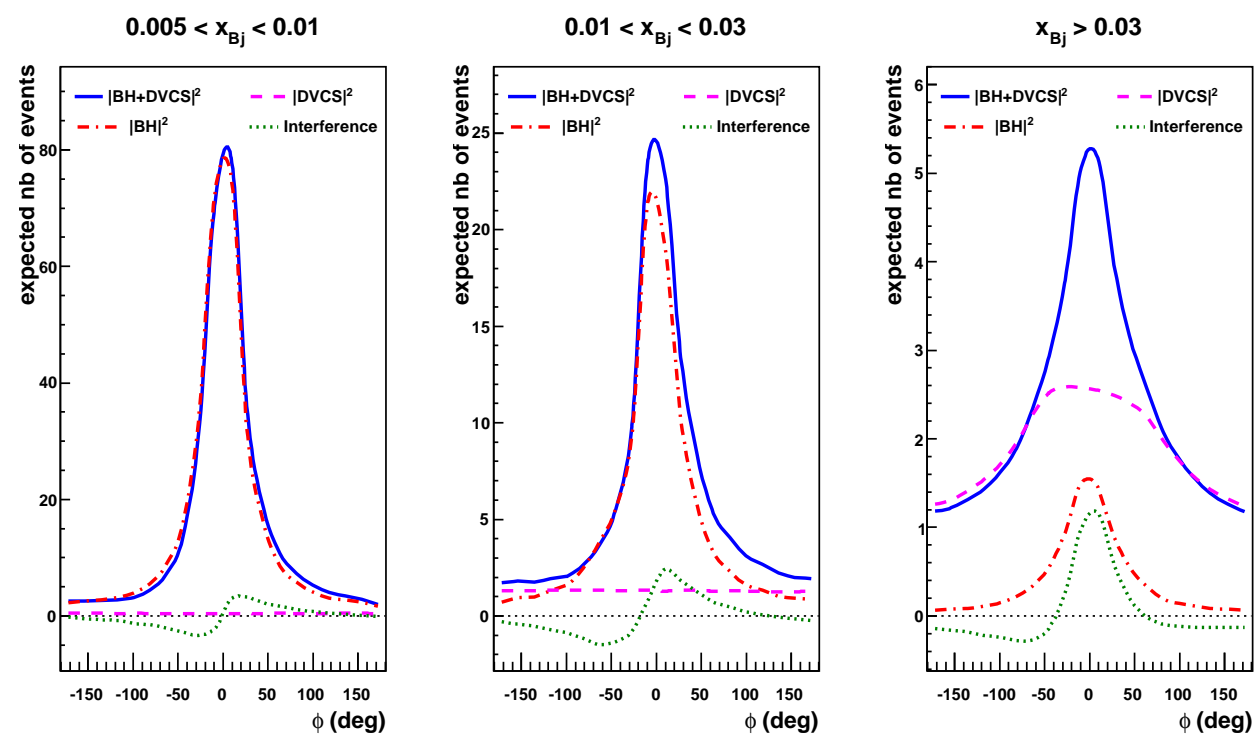

FIGURE 2. Monte Carlo simulation of the exclusive process $\mu^{+} p \rightarrow \mu^{\prime+} p \gamma$ for $Q^{2}>1 \mathrm{GeV}^{2}$, showing the $\phi$ angle distribution for three bins in $x_{B}: 0.005<x_{B}<0.01$ (left), $0.01<x_{B}<0.03$ (middle) and $0.03<x_{B}$ (right). The event yield shown is normalised to the integrated luminosity of the 2009 DVCS test run. It is based on the acceptance of the presently existing COMPASS set-up.

where $I$ is the interference term of Eq. (1), $P_{\mu}$ is the beam polarization and $e_{\mu}$ its charge in units of the elementary charge. The DVCS amplitude can be expanded in $1 / Q$ beyond leading twist- 2 including all twist- 3 contributions [2]. The dependence on $\phi$, the azimuthal angle between lepton scattering plane and photon production plane, is a characteristic feature of the cross section. Integration over $\phi$ and/or analysis of the angular dependence in $\phi$ allows us to isolate specific contributions that are sensitive to different combinations of quark GPDs. Gluon GPDs enter in DVCS only beyond leading order in $\alpha_{s}$ (LO), analogous to DIS.

At the CERN SPS M2 beamline, the 'natural' polarization of the muon beam produced from pion decay changes sign when the beam charge is reversed. Hence with the same apparatus the COMPASS experiment can perform separate measurements for the two beam charge/polarization states $\underset{\leftarrow}{\leftarrow}$ and $\rightrightarrows$, which can be used to calculate:

the 'Beam Charge (C) and Spin (S) Difference' (for Unpolarized $(U)$ proton target)

$$
\begin{aligned}
\mathscr{D}_{U, C S} & \equiv d \sigma^{\ddagger}-d \sigma^{\bar{\rightarrow}}=2\left[P_{\mu} d \sigma_{p o l}^{D V C S}+e_{\mu} \operatorname{Re} I\right] \\
& \propto\left(\left\{s_{1}^{D V C S} \sin \phi\right\}\right)+\left(c_{0}^{I}+c_{1}^{I} \cos \phi+\left\{c_{2}^{I} \cos 2 \phi+c_{3}^{I} \cos 3 \phi\right\}\right)
\end{aligned}
$$

in which the pure BH contribution cancels out. The coefficients $c_{i}^{I, D V C S}$ and $s_{i}^{I, D V C S}$ are related to certain combinations of Compton Form Factors (CFFs). A CFF $\mathscr{F}$ is a sum over flavors $f$, of convolutions of the respective GPDs $F^{f}$ with a perturbatively calculable kernel describing the hard $\gamma^{*} q$ interaction. Note that the contributions shown between a pair of braces corresponds to higher-twist or higher-order effects. The analysis of the $\phi$-dependence of the beam charge and spin difference $\mathscr{D}_{U, C S}$ will provide via the term $\operatorname{Re} I$ the two leading twist-2 expansion coefficients $c_{0}^{I}$ and $c_{1}^{I}$, the dominant contribution to which is related to the real part of the Compton form factor $\mathscr{H}$ that is in LO given by a flavor sum of convolutions involving the GPDs $\mathrm{H}^{f}$.

The 'Beam Charge and Spin Sum' of cross sections can also be evaluated:

$$
\begin{aligned}
\mathscr{S}_{U, C S} & \equiv d \sigma^{亡}+d \sigma^{\bar{\rightarrow}}=2\left[d \sigma^{B H}+d \sigma_{\text {unpol }}^{D V C S}+e_{\mu} P_{\mu} \operatorname{Im} I\right] \\
& \propto 2\left[d \sigma^{B H}\right]+\left(c_{0}^{D V C S}+\left\{c_{1}^{D V C S} \cos \phi+c_{2}^{D V C S} \cos 2 \phi\right\}\right)+\left(s_{1}^{I} \sin \phi+\left\{s_{2}^{I} \sin 2 \phi\right\}\right)
\end{aligned}
$$

in which the BH contribution does not cancel out. 1) The analysis of the $\phi$-dependence of $\mathscr{S}_{U, C S}$ will provide via the term $\operatorname{Im} I$ the leading twist-2 quantity $s_{1}^{I}$. Its dominant contribution is related to the imaginary part of the Compton 

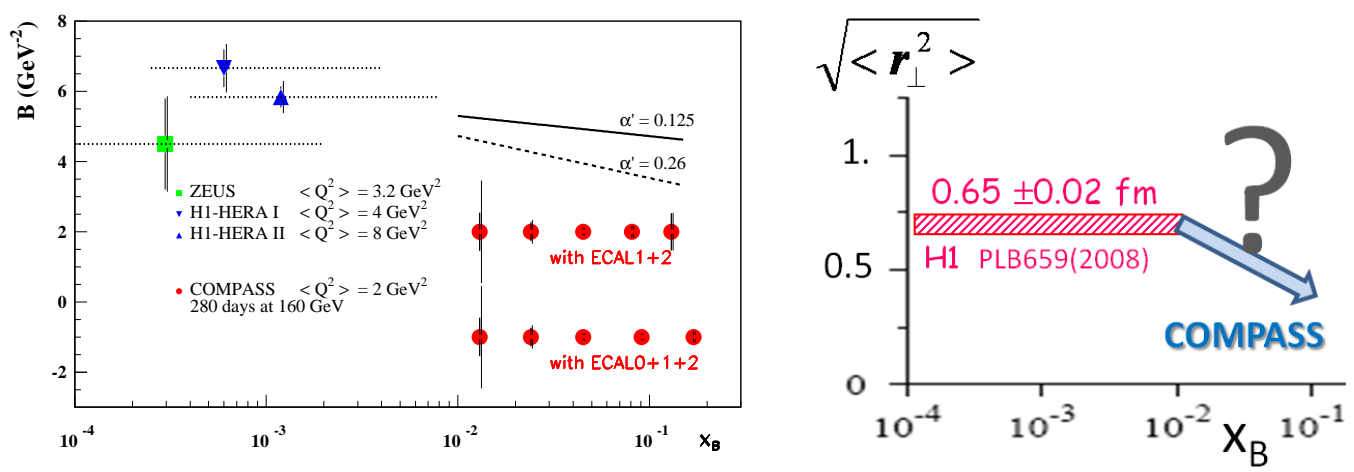

FIGURE 3. Left: Projections for measuring the $x_{B}$ dependence of the $t$-slope parameter $B\left(x_{B}\right)$ of the DVCS cross section, calculated for $1<Q^{2}<8 \mathrm{GeV}^{2}$. For comparison some HERA results with similar $\left\langle Q^{2}\right\rangle$ are shown [6,9]. The left vertical bar on each data point indicates the statistical error only while the right one includes also the systematic uncertainty, using only ECAL1 and ECAL2 (first row) and also ECAL0 (second row). Two different parameterisations are shown using $\alpha^{\prime}=0.125 \mathrm{GeV}^{-2}$ and $0.26 \mathrm{GeV}^{-2}$. Right: Transverse proton radius as a function of $x_{B}$. New and significant information will be obtained in the uncharted $x_{B}$ region, further elucidating the issue of "nucleon tomography".

form factor $\mathscr{H}$. 2) A parallel analysis can be performed subtracting the BH contribution when it is not too large, and integrating over $\phi$ to get rid of the complete interference term and of the $\phi$-dependent terms of the DVCS contribution. Thus the DVCS leading twist-2 quantity $c_{0}^{D V C S}$ can be isolated and its characteristic $t$-slope can be determined as a function of $x$, from which conclusions can be drawn on the transverse size of the nucleon over the $x$-range accessible to COMPASS in what is know as 'nucleon tomography'.

\section{Shrinkage of the nucleon size}

Using the $\phi$-integrated beam charge and spin sum after BH subtraction, figure 3 (left) shows the projected statistical accuracy for a measurement at COMPASS of the $x$-dependence of the $t$-slope parameter $B(x)$ of the DVCS cross section $\frac{d \sigma}{d t}(x) \propto \exp \left(-B(x)\right.$ t). In the simple ansatz $B(x)=B_{0}+2 \alpha^{\prime} \log \left(\frac{x_{0}}{x}\right)$, the shrinkage parameter $\alpha^{\prime}$ is known a long time to describe the decrease in nucleon size with increasing $x$. More recently, this ansatz was also used for the 'reggeized' description of a correlated $(x, t)$ dependence of GPDs. The $t$-slope of the GPD $H^{f}$ at a given $x$ was shown [4] to be related to the average impact parameter $\left\langle\left(b_{\perp}^{f}\right)^{2}\right\rangle$ in the distribution of partons of flavor $f$ carrying the longitudinal momentum fraction $x$ at a given $t: B^{f}(x) \sim 1 / 2\left\langle\left(b_{\perp}^{f}\right)^{2}\right\rangle(x)$.

Data on $B$ exist only for the HERA collider $x$-range from $10^{-4}$ to $0.01[6,9]$, below the COMPASS range $0.01<x<0.1$. In Fig. 3 only HERA results are reported for which the mean value $\left\langle Q^{2}\right\rangle$ is in the investigated domain by COMPASS. In the valence region, where no experimental determinations of $B$ exist, some information comes from fits adjusted to form factor data which give $\alpha^{\prime} \simeq 1 \mathrm{GeV}^{2}[10,11]$. For the low- $x$ sector, H1 results on $\alpha^{\prime}$ from exclusive $J / \psi$ production [7], which involves the generalized gluon distribution, are smaller by two standard deviations in the total experimental uncertainty than the corresponding value $\alpha^{\prime}=0.25$ for Pomeron exchange in soft scattering processes. For the simulation shown in figure 3 we chose the value $\alpha^{\prime}=0.125$.

This measurement will yield new and significant information in the context of 'nucleon tomography' as it is expected in chiral-dynamics approach [8]. In this approach, the gluon density is generated by the 'pion cloud' of the nucleon so that a significant increase in the transverse size of the nucleon is predicted for $x$ below the ratio of pion and proton masses, $m_{\pi} / m_{p} \approx 0.15$ which lies in the COMPASS domain. 


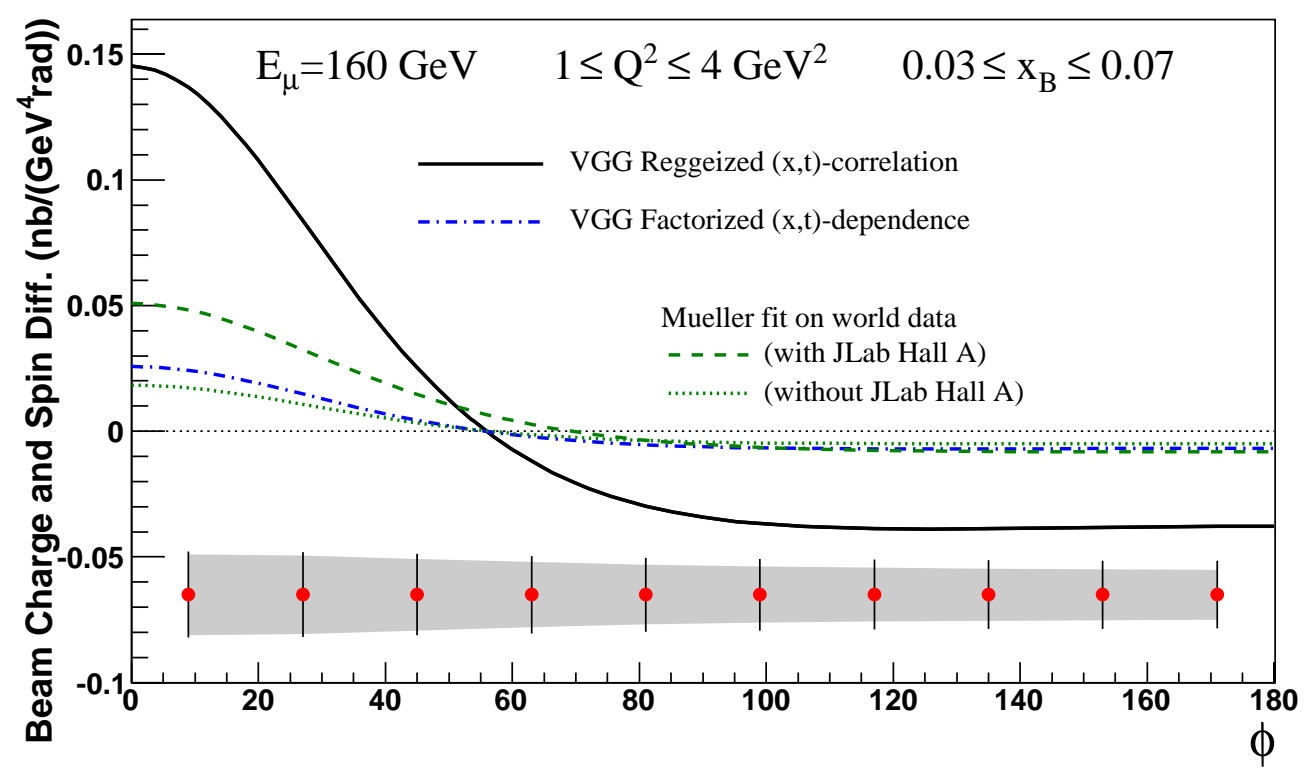

FIGURE 4. Projected statistical (error bars) and systematic (grey band) accuracy for a measurement of the $\phi$ dependence of the Beam Charge and Spin Difference at COMPASS for $0.03 \leq x \leq 0.07$ and $1 \leq Q^{2} \leq 4 \mathrm{GeV}^{2}$ and for 280 days of running time with a $2.5 \mathrm{~m} \mathrm{LH} 2$ target, an intensity of $4.6 \times 10^{8} \mu$ in a $48 \mathrm{~s}$ SPS spill period and an overall global efficiency $\varepsilon_{\text {global }}=0.1$. The solid and dash-dotted curves correspond to different variants of the VGG model [3] while the dashed and dotted curves show predictions based on first fits on world data [5].

\section{Beam Charge and Spin Asymmetry and sensitivity to models}

Using the beam charge and spin asymmetry $\mathscr{D}_{U, C S} / \mathscr{S}_{U, C S}$, figure 4 shows the projected statistical accuracy in a particular $\left(x, Q^{2}\right)$ bin, for a measurement of its $\phi$-dependence. Two of the curves are calculated using the 'VGG' GPD model [3]. As this model is meant to be applied mostly in the valence region, typically the value $\alpha^{\prime}=0.8$ is used in the 'reggeized' parameterization of the correlated $(x, t)$ dependence of GPDs. For comparison, also the model result for the 'factorized' $x, t$ dependence is shown, which corresponds to $\alpha^{\prime} \approx 0.1$ in the 'reggeized' ansatz.

A recent theoretical development exploits dispersion relations for Compton form factors. In this context, the additional curve is the result of a fitting procedure [5] including next-to-next-to leading order (NNLO) corrections which was developed and successfully applied to describe DVCS observables from very small values of $x$, for the HERA collider to large $x$ for HERMES and JLab.

\section{OBSERVATION OF EXCLUSIVE PHOTON PRODUCTION IN 2008 AND 2009 TEST BEAM DATA}

A preliminary study of the feasibility of DVCS measurements was performed in 2008 and 2009 with minimum disturbance of the ongoing hadron spectroscopy measurements. The experimental set-up makes use of the $40 \mathrm{~cm}$ long $\mathrm{LH}_{2}$ target, surrounded by a Recoil Proton Detector (RPD). It includes all detectors presently available including the ECAL1 and ECAL2 electromagnetic calorimeters for photon detection. Apart from the shorter target and the corresponding RPD lengths, this set-up has the principal features of the one foreseen for the proposed future GPD program. DVCS test measurements were performed using both $\mu^{+}$and $\mu^{-}$beams of $160 \mathrm{GeV}$ energy, with an intensity of about $1 / 3$ of the maximum in 2008 and close to the maximum intensity in 2009 . The 2008 DVCS test data allowed one to clearly identify exclusive photon production and to obtain a first direct determination of the global overall efficiency. The latter, including also SPS beam and spectrometer availabilities, trigger and DAQ efficiencies, was found to be close to the value of $10 \%$ assumed for the projections. The 2009 DVCS tests data provided a first direct measurement of the relative contributions of the BH and DVCS in the $x_{B}$ domain available at COMPASS (Fig. 5). Note that the predicted $\phi$ dependence of the DVCS cross section is not flat in $\phi$ due to acceptance effects in the 2009 

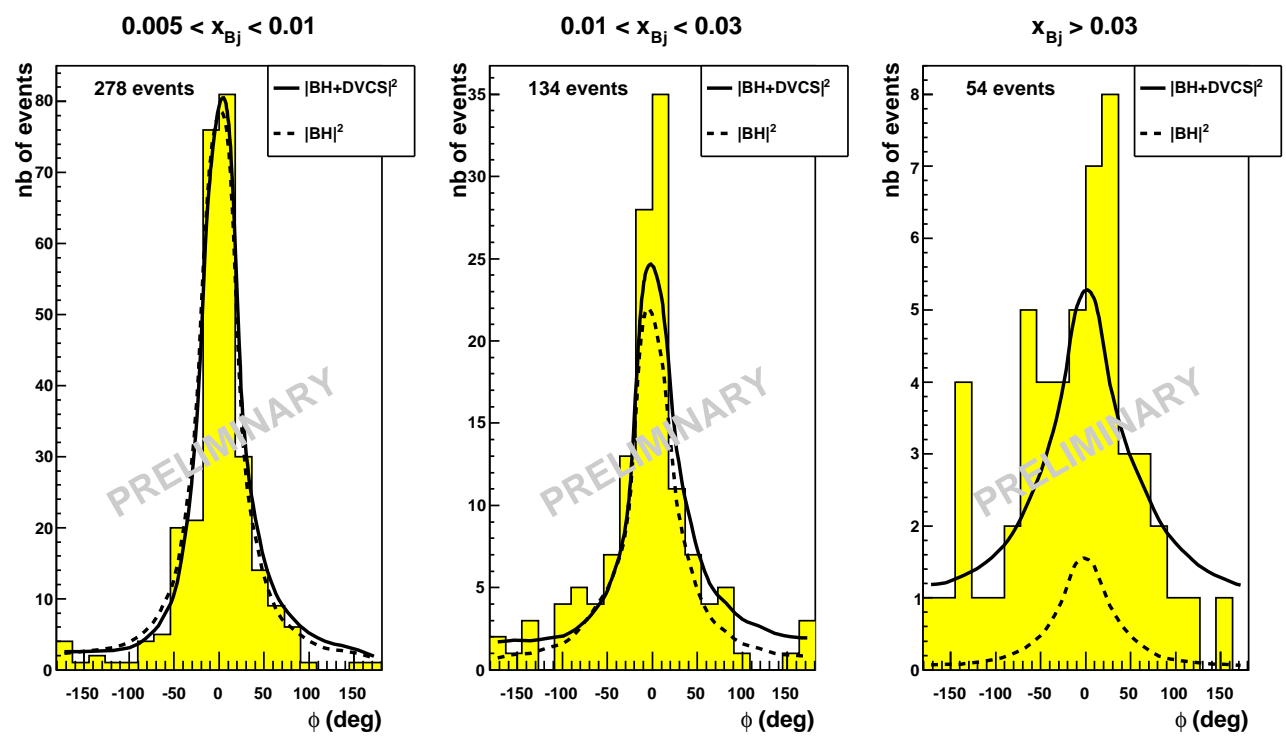

FIGURE 5. Distribution in the azimuthal angle $\phi$ for measured exclusive single-photon events, $\mu p \rightarrow \mu^{\prime} p \gamma$ with $Q^{2}>1 \mathrm{GeV}^{2}$, in the same three $x_{B}$ bins as in Fig. 1. Shown here is a Monte Carlo simulation of only the BH process and of both the BH and DVCS processes.

set-up as there is no electromagnetic calorimeter at large photon angles. This will be improved by the addition of the new calorimeter ECAL0 already mentioned above.

\section{REFERENCES}

1. COMPASS-II proposal, SPSC-P-340,

http://cdsweb.cern.ch/record/1265628/files/SPSC-P-340.pdf, May 17, 2010

2. A.V. Belitsky, D. Müller and A. Kirchner, Nucl. Phys. B 629 (2002) 323.

3. M. Vanderhaeghen, P.A.M. Guichon and M. Guidal, Phys. Rev. Lett. 80 (1998) 5064; Phys. Rev. D 60 (1999) 094017; K. Goeke, M.V. Polyakov and M. Vanderhaeghen, Prog. Part. Nucl. Phys. 47 (2001) 401.

4. M. Burkardt, Phys. Rev. D 62 (2000) 071503; erratum-ibid. d 66 (2002) 119903;

5. K. Kumericki,D. Mueller and K. Passek-Kumericki, Nucl. Phys. B 794 (2008) 244, K. Kumericki and D. Mueller, arXiv 0904.0458[hep-ph]

6. H1, A. Aktas et al., Eur. Phys. J.C 44 (2005) 1, F.D. Aaron et al., Phys. Lett. B 659 (2008) 796.

7. H1, A. Aktas et al., Eur. Phys. J.C 46 (2006) 585.

8. M. Strikman and C. Weiss, Phys. Rev. D69 (2004) 054012.

9. ZEUS, S. Chekanov et al., DESY-08-178, arXiv:hep-exp:0812.2517v3.

10. M. Diehl, Th. Feldmann, R. Jakob and P. Kroll, Eur. Phys. J. C 39 (2005) 1.

11. M. Guidal, M.V. Polyakov, A.V. Radyushkin and M. Vanderhaeghen, Phys. Rev. D 72 (2005) 054013. 